\title{
RADIALLY SYMMETRIC SOLUTIONS OF A CHEMOTAXIS MODEL IN THE PLANE - THE SUPERCRITICAL CASE
}

\author{
PIOTR BILER \\ Instytut Matematyczny, Uniwersytet Wroctawski \\ pl. Grunwaldzki 2/4, 50-384 Wroctaw, Poland \\ E-mail:Piotr.Biler@math.uni.wroc.pl
}

\begin{abstract}
The existence, uniqueness and large time behaviour of radially symmetric solutions to a chemotaxis system in the plane $\mathbb{R}^{2}$ are studied for the (supercritical) value of mass greater than $8 \pi$.
\end{abstract}

1. Introduction. This paper, a sequel of $[5,6]$, deals with the nonlinear, nonuniformly parabolic problem on $(0, \infty) \times(0, \infty)$

$$
M_{t}=4 s M_{s s}+\frac{1}{\pi} M M_{s}
$$

together with the boundary (at 0 and $\infty$ ) and initial conditions

$$
\begin{gathered}
M(0, t)=0, \quad M(\infty, t) \equiv \lim _{s \rightarrow \infty} M(s, t)=\widehat{M}, \\
M(s, 0)=M_{0}(s), \quad s \in(0, \infty),
\end{gathered}
$$

with a nondecreasing continuous function $M_{0}$ on $(0, \infty)$. The motivations to study that problem stem from a simplified chemotaxis model of Keller-Segel considered in the whole plane $\mathbb{R}^{2}$, cf. e.g. $[9,6]$, and a model of self-attracting particles considered in $[8,4,7]$, consisting of equations

$$
\begin{aligned}
u_{t} & =\nabla \cdot(\nabla u+u \nabla \varphi), \quad(x, t) \in \mathbb{R}^{2} \times(0, \infty), \\
\varphi & =E_{2} * u, \quad(x, t) \in \mathbb{R}^{2} \times(0, \infty) .
\end{aligned}
$$

Here, $u=u(x, t) \geq 0$ denotes the density of microorganisms (e.g. amoebae), $\varphi=\varphi(x, t)$ the concentration of a chemoattractant secreted by themselves, and $E_{2}(z)=\frac{1}{2 \pi} \log |z|$

2000 Mathematics Subject Classification: 35Q, 35K60, 35B40.

Key words and phrases: chemotaxis system, infinite domain, radially symmetric solutions, supercritical case.

The paper is in final form and no version of it will be published elsewhere. 
the fundamental solution of the Laplacian in $\mathbb{R}^{2}$, so that $\varphi$ is actually a solution to the Poisson equation $\Delta \varphi=u$. For radially symmetric solutions, introducing the mass distribution function

$$
M(s, t) \equiv \int_{B(0, \sqrt{s})} u(x, t) \mathrm{d} x, \quad s \in[0, \infty),
$$

the previous parabolic-elliptic system for $(u, \varphi)$ reduces to the single parabolic equation (1.1) for the transformed 'cumulated mass' variable $M$ with $\widehat{M}=\left\|u_{0}\right\|_{L^{1}}$. The conservation of total mass is interpreted as the preservation of the boundary condition (1.2) at infinity. The simplified, radially symmetric problem gives a deeper insight into the asymptotic behavior of solutions for large time, cf. [9] for the self-similar asymptotics of general solutions of (1.4)-(1.5) in the subcritical case, and [6] for the radially symmetric case.

The equation (1.1) features a nonuniform diffusion described by the term $4 s M_{s s}$ and a simple nonlinear convection term $M M_{s} / \pi$, and the dynamics of $M$ is governed by the competition between these two terms. There is actually a threshold value of $\widehat{M}(\widehat{M}=8 \pi)$ such that, roughly speaking, the diffusion term prevails for $\widehat{M}$ below $8 \pi$ (and leads to mass spreading to infinity), while the nonlinearity (pushing to aggregation of mass in the original chemotaxis problem) is more important for $\widehat{M}>8 \pi$. In fact, it is well-known by now that solutions to (1.4), (1.5) blow up in finite time if $\widehat{M}=\left\|u_{0}\right\|_{L^{1}}>8 \pi$ (see, e.g., $[10,3,4,12])$ and that the condition $\widehat{M}=\left\|u_{0}\right\|_{L^{1}}<8 \pi$ is a sufficient condition for the existence of global in time solutions: see $[12,4]$ for radially symmetric solutions and [9] for the general case which was recently solved with the help of the optimal logarithmic Hardy-Littlewood-Sobolev inequality. The subcritical and critical cases $\widehat{M} \leq 8 \pi$ have been recently studied in [6]. First of all, we proved in [6, Th. 2.1] the well-posedness of the problem (1.1)-(1.3). That is: solutions of that problem exist, are unique and enjoy local stability property as a consequence of the $L^{1}$-contractivity property for the diffusionconvection equations, [6, Lemma 2.2].

Second, the boundary condition at $s=0$ is either satisfied classically whenever $\widehat{M}<$ $8 \pi$ ([6, Prop. 4.2]) or is satisfied a.e. in $t \in(0, \infty)$ if $\widehat{M}=8 \pi$.

Finally, compared to relatively simple time asymptotics for the equation (1.1) considered on the finite interval $s \in(0,1)$ in [5], the picture for solutions of $(1.1)$ for $s \in(0, \infty)$ is much more delicate. The balance between diffusion and convection leads to the existence of steady states $M_{b}(s)=8 \pi s /(s+b), b>0$. These steady states are locally asymptotically stable in $L_{\mathrm{loc}}^{1}(0, \infty)$ but $\left\|8 \pi-M_{b}\right\|_{L^{1}}=\infty$ for each $b>0$. Moreover, if $M$ is a solution to (1.1)-(1.3), then $\|8 \pi-M(t)\|_{L^{1}}=\left\|8 \pi-M_{0}\right\|_{L^{1}}$ for $t \geq 0$. Consequently, the set $\left\{M_{b}: b>0\right\}$ cannot attract the solutions starting from an initial datum in $8 \pi+L^{1}(0, \infty)$.

The degeneracy of the diffusion term $4 s M_{s s}$ does not guarantee a priori that the required boundary condition at $s=0$ is satisfied. In fact, for $\widehat{M}>8 \pi$ there occurs a phenomenon of lost of the boundary condition at $s=0$. To the best of our knowledge, the proofs of blow up of solutions for the original chemotaxis system showed only that $\|u(t)\|_{L^{\infty}} \rightarrow \infty$ as $t \nearrow T$ for some $T<\infty$, so that $\left\|M_{s}(., t)\right\|_{L^{\infty}} \rightarrow \infty$ as $t \nearrow T$, cf. [2]. Note that the formulations (1.1)-(1.2) and (1.4)-(1.5) for the radially symmetric solutions are not equivalent because of the different regularity of $M$ and $u$ required in order to have 
(weak, mild, classical) solutions of those problems. In particular, $M$ that does not satisfy the boundary condition at $s=0$ corresponds to a measure valued solution $u=u(t)$. The theory of such measure valued solutions is not completely satisfactory up to now, cf. [9], [15], [1]. Concentration phenomena for (1.4)-(1.5) are thoroughly studied in [16].

Here, we will show that not only the derivative $M_{s}$ blows up but also $M^{*}(t) \equiv$ $\lim _{s \rightarrow 0} M(s, t)$ jumps from $M^{*}(t)=0$ for (a.e.) $0 \leq t \leq T$ to $M^{*}(T)=8 \pi$, and then $M^{*}(t)$ is strictly increasing with $\lim _{t \rightarrow \infty} M^{*}(t)=\widehat{M}$. To prove this we will use suitable exploding subsolutions for the problem (1.1)-(1.3). Some formal results in this direction have been announced in the preprint [14] but even the local in time existence of solutions of (1.1)-(1.3) has not been described in detail in [14]. Here, we combine the approximation techniques from $[5,6]$ with a construction of suitable local in time classical supersolutions for the problem (1.1)-(1.3). Then, the asymptotics of solutions is described using essentially the ideas in [14] and [7].

The results obtained here are explicit examples of the lost of a boundary condition for solutions of an initial-boundary value problem for a nonlinear, nonuniformly parabolic equation. This phenomenon is a bit different from the related analysis of linear nonuniformly parabolic boundary value problems as studied in the framework of the famous Feller test analysis, cf. [13, Ch. II, Th. 2.6] and [8, the proof of Th. 1, (iv)]. The main difference is that for the nonlinear problem the boundary condition is lost after some time while for the linear problems solutions either exist and satisfy the Dirichlet boundary condition or they cannot satisfy such a boundary condition, and they simply do not exist, even locally in time.

Finally, note that a more general problem (the so-called 'star problem' in [8]) consisting of (1.1) and (1.3) with the conditions $M(0, t)=m^{*}, M(\infty, t)=\widehat{M}$, for $m^{*} \in[0,4 \pi)$, can be treated by similar methods. Its solutions behave for $\widehat{M}<8 \pi-m^{*}$ subcritically, and for $\widehat{M}>8 \pi-m^{*}$ supercritically.

2. The auxiliary problem. In this section the equation (1.1) is considered on the bounded space interval. The results on the existence, uniqueness and comparison of solutions will be used in the next section to obtain related properties of solutions of (1.1)-(1.3) on the whole half-line $(0, \infty) \ni s$.

Since the equation (1.1) is invariant under the scaling $s \mapsto R s, t \mapsto R t$ for each $R>0$, it suffices to solve the problem on the finite space interval for $R=1$ only. Thus, we consider the problem

$$
\begin{aligned}
M_{t} & =4 s M_{s s}+\frac{1}{\pi} M M_{s}, \\
M(0, t) & =0, \quad M(1, t)=\widehat{M}, \\
M(s, 0) & =M_{0}(s), \quad s \in(0,1),
\end{aligned}
$$

and prove the following

Proposition 2.1. Let $\widehat{M}>0$ and $M_{0, s}(0)<\infty$. There exists time $T>0$ and a unique function $M \in \mathcal{C}\left([0, T) ; L^{2}(0,1)\right) \cap \mathcal{C}_{s, t}^{2,1}((0,1) \times(0, T))$ such that

$$
0 \leq M(s, t) \leq \widehat{M}, \quad M_{s}(s, t) \geq 0 \text { for }(s, t) \in(0,1) \times(0, T),
$$




$$
\begin{aligned}
& M^{*}(t) \equiv \inf _{s \in(0,1)} M(s, t)=0 \text { for each } t \in(0, T), \\
& M(1, t)=\widehat{M} \text { for each } t \in(0, T),
\end{aligned}
$$

and $M$ satisfies the equation (2.1) for $(s, t) \in(0,1) \times(0, T)$, together with the initial condition (2.3). The solutions of the problem (2.1)-(2.3) satisfy the comparison principle.

Proof. Observe first that for $\widehat{M} \leq 8 \pi$ we have the global in time existence of classical solutions: $T=\infty$. If $\widehat{M}<8 \pi$, then the assumption $M_{0, s}(0)<\infty$ is even superfluous. However, without that assumption if $\widehat{M}=8 \pi$ we may conclude that $M^{*}(t)=0$ only a.e. in $(0, \infty)$. The proofs in the subcritical case can be found in [5, Th. 2.1, Prop. 2.5] and in [6, Prop. 4.2]. So, in the sequel, we assume that $\widehat{M}>8 \pi$ and show the local in time existence of solutions to (2.1)-(1.3).

Existence. The scheme of the proof is similar to that in [6, Th. 2.1] for the subcritical case. However, we need another tool to control the behavior of $M$ near $s=0$ since the lost of the boundary condition at $s=0$ is expected for $t$ sufficiently large. This will be done using a suitable local in time supersolution of the regularized problem (2.1)-(2.3).

Consider for $\varepsilon \in(0,1)$ and any $T_{0} \in(0, \infty)$ the uniformly parabolic initial-boundary value problem

$$
\begin{aligned}
M_{\varepsilon, t} & =4(s+\varepsilon) M_{\varepsilon, s s}+\frac{1}{\pi} M_{\varepsilon} M_{\varepsilon, s}, \quad(s, t) \in(0,1) \times\left(0, T_{0}\right), \\
M_{\varepsilon}(0, t) & =\widehat{M}-M_{\varepsilon}(1, t)=0, \quad t \in\left(0, T_{0}\right), \\
M_{\varepsilon}(s, 0) & =M_{0, \varepsilon}(s), \quad s \in(0,1),
\end{aligned}
$$

with $M_{0, \varepsilon} \in H^{1}(0,1)$ approximating $M_{0}$ so that $0 \leq M_{0}(s)-M_{0, \varepsilon}(s) \leq \varepsilon$. This problem has (global in time) solutions $M_{\varepsilon} \in \mathcal{C}\left([0,1] \times\left[0, T_{0}\right)\right) \cap \mathcal{C}_{s, t}^{2,1}\left((0,1) \times\left(0, T_{0}\right)\right)$ for each $T_{0}>0$. By a standard application of the parabolic regularity theory in [11, Th. III.10.1, IV.10.1]

$$
\left\|M_{\varepsilon}\right\|_{\mathcal{C}_{s, t}^{2+\alpha, 1+\alpha}\left([\delta, 1] \times\left[\tau, T_{0}\right]\right)} \leq C\left(\alpha, \delta, \tau, T_{0}\right)
$$

for each $\tau \in\left(0, T_{0}\right)$ and $\alpha \in(0,1)$, with a positive constant $C\left(\alpha, \delta, \tau, T_{0}\right)$ depending on $\alpha, \delta, \tau$ and $T_{0}$ but independent of $\varepsilon \in(0,1)$.

If $\widehat{M}>8 \pi, M_{0, s}(0)<\infty$, the behavior of $M_{\varepsilon}$ can be controlled uniformly in $\varepsilon \in(0,1)$ locally in time only. Indeed, for any $A>\widehat{M}>8 \pi$ there exists a sufficiently small $\mathcal{T}_{0}>0$ so that

$$
M_{0}(s) \leq \frac{A s}{s+\left(\frac{A}{\pi}-8\right) \mathcal{T}_{0}} .
$$

Next, for $\varepsilon \in(0,1)$ the function

$$
\bar{M}_{\varepsilon}(s, t)=\frac{A(s+\varepsilon)}{(s+\varepsilon)+\left(\frac{A}{\pi}-8\right)\left(\mathcal{T}_{0}-t\right)}
$$

is a supersolution of the problem $(2.7)-(2.9)$ on $(0,1) \times\left(0, \mathcal{T}_{0}\right)$. Indeed, since that problem is uniformly parabolic, so if we define $L_{\varepsilon} w=w_{t}-4(s+\varepsilon) w_{s s}-\frac{1}{\pi} w w_{s}$, then

$$
\begin{aligned}
L_{\varepsilon} \bar{M}_{\varepsilon}= & \frac{A}{(s+\varepsilon+b(t))^{3}}(-\dot{b}(t)(s+\varepsilon)(s+\varepsilon+b(t)) \\
& \left.+8(s+\varepsilon) b(t)-\frac{A}{\pi}(s+\varepsilon) b(t)\right) \geq 0,
\end{aligned}
$$


where $b(t)=\left(\frac{A}{\pi}-8\right)\left(\mathcal{T}_{0}-t\right)$ so that $\dot{b}(t)=-\left(\frac{A}{\pi}-8\right)$ and $\bar{M}_{\varepsilon}(s, 0) \geq M_{0}(s), \bar{M}_{\varepsilon}(1, t) \geq$ $M_{0}(1)$. Using $\bar{M}(s, t)=\lim _{\varepsilon \rightarrow 0} \bar{M}_{\varepsilon}(s, t)=\inf _{\varepsilon \rightarrow 0} \bar{M}_{\varepsilon}(s, t)=\frac{A s}{s+\left(\frac{A}{\pi}-8\right)\left(\mathcal{T}_{0}-t\right)}$ and $(2.10)$ we can pass to the limit $\varepsilon \rightarrow 0$ in $(2.7)-(2.9)$ on the whole interval $(0,1)=(0, \delta] \cup[\delta, 1)$ and $t \in\left(0, \mathcal{T}_{0}\right)$. Indeed, $\bar{M}$ controls all the approximations $\bar{M}_{\varepsilon}$ on $[0, \delta] \times\left[0, \mathcal{T}_{0}\right)$, and $(2.10)$ permits us to pass to the limit $\varepsilon \rightarrow 0$ using the Ascoli-Arzelà compactness argument as was in [5, Th. 2.1]. Thus, there exists a function $M$ satisfying (2.4), (2.5), (2.6) at least for $0<t<T=\mathcal{T}_{0}$. Using again (2.10) we conclude that the derivatives of $M$ exist and (2.1) is satisfied.

It is important to observe that $M_{s}(0, t)=\sup _{s>0} M_{s}(s, t)<\infty$ for each $t<\mathcal{T}_{0}$, i.e. as long as the behavior of $\lim _{\varepsilon \rightarrow 0} M_{\varepsilon}(s, t)$ is controlled by $\bar{M}(s, t)$ together with $(2.10)$.

Note that Lemma 2.3 in [5] is valid for the problem (2.1)-(2.3) with sufficiently small $T>0$, and this identifies the initial condition for $M$, and conclude that $M \in$ $\mathcal{C}\left([0, T), L^{2}(0,1)\right)$ exactly as was in $[5]$.

Uniqueness. This property follows from the $L^{1}$-contraction property typical for convection-diffusion equations. We consider, similar to [5, Th. 3.1], the difference $N=M-\bar{M}$ of two solutions $M$ and $\bar{M}$ which satisfies the equation

$$
N_{t}=\frac{\partial}{\partial s}\left(4 s N_{s}+\frac{1}{2 \pi} N(M+\bar{M}-8 \pi)\right)
$$

with $N(0, t)=N(1, t)=0$ for all $t \in(0, T)$. For $\delta \in(0,1)$ and $r \in \mathbb{R}$, we use a convex approximation of $r \mapsto|r|$, e.g.,

$$
\Phi_{\delta}(r) \equiv\left\{\begin{array}{cl}
\frac{1}{\delta}\left(|r|-\frac{\delta}{2}\right)^{2} & \text { if } \quad|r| \in[0, \delta], \\
|r|-\frac{3}{4} \delta & \text { if } \quad|r| \in(\delta, \infty) .
\end{array}\right.
$$

We multiply $(2.13)$ by $\Phi_{\delta}^{\prime}(N)$ and integrate over $(0,1)$ to obtain

$$
\begin{aligned}
\frac{\mathrm{d}}{\mathrm{d} t} \int_{0}^{1} \Phi_{\delta}(N) d s= & \left.4 s N_{s} \Phi_{\delta}^{\prime}(N)\right|_{0} ^{1}+\left.\frac{1}{2 \pi} \Phi_{\delta}^{\prime}(N) N(M+\bar{M}-8 \pi)\right|_{0} ^{1} \\
& -\int_{0}^{1} 4 s \Phi_{\delta}^{\prime \prime}(N) N_{s}^{2} \mathrm{~d} s-\frac{1}{2 \pi} \int_{0}^{1} \Phi_{\delta}^{\prime \prime}(N) N_{s} N(M+\bar{M}-8 \pi) \mathrm{d} s \\
\leq & -\frac{1}{2 \pi} \int_{0}^{1} \Phi_{\delta}^{\prime \prime}(N) N_{s} N(M+\bar{M}-8 \pi) \mathrm{d} s .
\end{aligned}
$$

Observe that $N_{s}$ belongs to $L^{\infty}\left((0, \infty) ; L^{1}(0,1)\right), M, \bar{M}$ and $N$ are bounded, and $r \mapsto$ $r \Phi_{\delta}^{\prime \prime}(r)$ is bounded and converges a.e. towards zero as $\delta \rightarrow 0$. Thus, the Lebesgue dominated convergence theorem ensures that the right-hand side of the above inequality converges to zero as $\delta \rightarrow 0$, from which we conclude that $t \mapsto\|M(t)-\bar{M}(t)\|_{L^{1}(0,1)}$ is a nonincreasing function of time $t \in(0, T)$.

It is important to recall here that the structure of (1.1) and the uniqueness of solutions of the initial-boundary value problem (2.1)-(2.3) imply the comparison principle for solutions, as was in [5, Sections 3, 4].

Now we study the blow up of solutions of the problem $(2.1)-(2.3)$ if $\widehat{M}>8 \pi$. This will be done constructing a suitable blowing up subsolution of that problem in the disc 
reminiscent of the function considered in the famous paper by W. Jäger and S. Luckhaus [10], see also [7, p. 358]. Then a continuation of the solution $M$ will be constructed for arbitrarily large times.

Proposition 2.2. The solution constructed in Proposition 2.1 can be continued to a unique function $M$ on $(0,1) \times(0, \infty)$ satisfying (2.1) pointwise in $(0,1) \times(0, \infty)$ and $M(0, t) \geq 0, M(1, t)=\widehat{M}$, (2.3). Moreover, if $M_{0, s}(0)>0$ then there exists $T>0$ such that $M^{*}(T)>0$.

Proof. Since the continuation of solutions is done in both cases $s \in(0,1)$ and $s \in(0, \infty)$ in a similar way, we postpone the proof to the next section where the original problem (1.1)-(1.3) will be studied.

For each $a \in(8 \pi, \widehat{M})$ and $\gamma>0$ (small enough) there exists $b>0$ such that

$$
\frac{a s}{s+b^{2}}+\gamma s \leq M_{0}(s) \text { for each } s \in(0,1) \text {. }
$$

However, there is a constant $c>0$, e.g. $c<\min \left(\frac{a}{\pi}-8, \frac{\gamma}{\pi}\right)$, such that

$$
\underline{M}(s, t)=\frac{a s}{s+(b-c t)^{2}}+\gamma s
$$

is a subsolution of the problem $(2.1),(2.3)$. Formally, if $L w=w_{t}-4 s w_{s s}-\frac{1}{\pi} w w_{s}$, then we have

$$
L \underline{M} \leq \frac{a s}{(s+b(t))^{3}}\left(-\dot{b}(t)(s+b(t))-\left(\frac{a}{\pi}-8\right) b(t)-\frac{\gamma}{\pi}(s+b(t))^{2}\right) \leq 0
$$

for $b(t)=(b-c t)^{2},(s, t) \in(0,1) \times(0, \mathcal{T})$ with $\mathcal{T}=\frac{b}{c}$. Therefore $M(s, t) \geq \underline{M}(s, t)$ loses its boundary condition at $s=0$ at a moment $t=T$ not later than $\mathcal{T}: T \leq \mathcal{T}$.

3. The main result. We prove in this section a theorem on the solvability and loss of a boundary condition for solutions of the problem (1.1)-(1.3) using the auxiliary construction in the preceding section.

THEOREM 3.1. Consider $\widehat{M}>8 \pi$ and a continuous nondecreasing function $M_{0}$ such that $M_{0}(0)=0,0<M_{0, s}(0)<\infty$ and $M_{0}(\infty)=\widehat{M}$. There exists time $T>0$ and a unique function $M \in \mathcal{C}\left([0, T) ; L_{\text {loc }}^{2}(0, \infty)\right) \cap \mathcal{C}_{s, t}^{2,1}((0, \infty) \times(0, T))$ such that

$$
\begin{aligned}
& 0 \leq M(s, t) \leq \widehat{M}, M_{s}(s, t) \geq 0 \text { for }(s, t) \in(0, \infty) \times(0, T), \\
& M^{*}(t) \equiv \inf _{s \in(0, \infty)} M(s, t)=0 \text { for } t \in(0, T),
\end{aligned}
$$

and $M$ satisfies the equation (1.1) for $(s, t) \in(0, \infty) \times(0, T)$ with the initial condition (1.3) and $M(\infty, t)=\widehat{M}, t \in(0, T)$. Moreover, there exists a continuation of the solution $M$ past $t=T$, still denoted by $M, M \in \mathcal{C}_{s, t}^{2,1}((0, \infty) \times(0, \infty))$ which satisfies the equation (1.1) for $(s, t) \in(0, \infty) \times(0, \infty)$. This continuation is such that $M^{*}(T) \geq 8 \pi$ and $M^{*}(t)$ is a strictly increasing function of $t \in(T, \infty)$ with $\lim _{t \rightarrow \infty} M^{*}(t)=\widehat{M}$.

Proof. Existence. The local in time solution $M$ is obtained in a way similar to that in [6, Th. 2.1] by approximating this with solutions $M=M_{R}$ of the initial-boundary value problems on $(0, R) \times(0, T), R \rightarrow \infty$,

$$
M_{t}=4 s M_{s s}+\frac{1}{\pi} M M_{s}
$$




$$
\begin{aligned}
& M(0, t)=0, \quad M(R, t)=M_{0}(R), \\
& M(s, 0)=M_{0}(s), \quad s \in(0, R) .
\end{aligned}
$$

The existence of solutions to the above problem is guaranteed by Proposition 2.1. Taking, for instance, $R=n, n \in \mathbb{N}, n \rightarrow \infty$, we are able to extract a subsequence of $\left\{M_{n}\right\}$ convergent to a function $M$ on each rectangle of the form $[\delta, \Delta] \times[\tau, T-\tau], 0<\delta<\Delta$, $\Delta<n, 0<\tau<T / 2$. Here $T>0$ can be chosen similarly to $T=\mathcal{T}_{0}$ in (2.11), in order to satisfy $M_{0}(s) \leq \frac{A s}{s+\left(\frac{A}{\pi}-8\right) \mathcal{T}_{0}}$ with a given $A>\widehat{M}>8 \pi$. That convergence is a consequence of the locally uniform Schauder estimates for $M_{n}$

$$
\left\|M_{n}\right\|_{\mathcal{C}_{s, t}^{2+\alpha, 1+\alpha}([\delta, \Delta] \times[\tau, T-\tau])} \leq C(\alpha, \delta, \Delta, \tau)
$$

for each $\alpha \in(0,1)$. Moreover, the behavior near $s=0$ for $t \in(0, T)$ is controlled: $M_{n}(s, t) \leq \bar{M}(s, t)$, where

$$
\bar{M}(s, t)=\frac{A s}{s+\left(\frac{A}{\pi}-8\right)\left(\mathcal{T}_{0}-t\right)}
$$

is defined by the same formula as was in the proof of Proposition 2.1 but for all $s \in(0, \infty)$. Of course, $\bar{M}$ is a supersolution for each of the problems (3.3)-(3.5) involving $M_{n}$.

The uniqueness of solutions $M \in \mathcal{C}\left((0, T) ; L_{\text {loc }}^{2}(0, \infty)\right) \cap \mathcal{C}_{s, t}^{2,1}((0, \infty) \times(0, T))$ is proved along the lines of the uniqueness proof in Proposition 2.1, the unboundedness of the space domain $(0, \infty) \ni s$ making no essential changes.

Blow up. Here we will use a stationary subsolution to compactify the domain, and then recall the construction of a blowing up subsolution in Section 2.

Observe that if $M_{0}(0)=0, M_{0}(\infty)=\widehat{M}>8 \pi$ and $M_{0, s}(0)>0$ (in fact, it suffices to have a bound $M_{0}(s) \geq \delta s$ for some $\delta>0$ and each $s$ in a neighborhood of 0 ), then for each $A \in(8 \pi, \widehat{M})$ there exists $B>0$ such that

$$
\underline{M}_{1}(s)=\frac{A s}{s+B} \leq M_{0}(s) \text { for each } s \in(0, \infty)
$$

is a (time independent) subsolution of the problem (1.1)-(1.3). Indeed, if $L w=w_{t}-$ $4 s w_{s s}-\frac{1}{\pi} w w_{s}$, then

$$
L \underline{M}_{1}=\frac{A B s}{(s+B)^{3}}\left(8-\frac{A}{\pi}\right) \leq 0,
$$

and therefore $\underline{M}_{1}(s) \leq M(s, t)$ by the comparison principle.

Next, let us note that for each $a \in(8 \pi, A)$ there exist $s_{0}>0$ and $\gamma>0$ such that $a+\gamma s_{0} \leq \frac{A s_{0}}{s_{0}+B}$. Moreover, there exists $b>0$ (we may diminish, if necessary, $\gamma>0$ ) such that $\frac{a s}{s+b^{2}}+\gamma s \leq M_{0}(s)$ for each $s \in\left(0, s_{0}\right)$. Thus, there is $c>0$, e.g. $c<\min \left(\frac{a}{\pi}-8, \frac{\gamma}{\pi}\right)$, such that

$$
\underline{M}(s, t)=\frac{a s}{s+(b-c t)^{2}}+\gamma s
$$

is a subsolution of the problem $(1.1)$ on $\left(0, s_{0}\right), M(s, 0)=M_{0}(s)$ for $s \in\left(0, s_{0}\right)$ and $M\left(s_{0}, t\right)=M_{0}\left(s_{0}\right)$. We have as in the proof of Proposition 2.2

$$
L \underline{M} \leq \frac{a s}{(s+b(t))^{3}}\left(-\dot{b}(t)(s+b(t))-\left(\frac{a}{\pi}-8\right) b(t)-\frac{\gamma}{\pi}(s+b(t))^{2}\right) \leq 0
$$

for $b(t)=(b-c t)^{2},(s, t) \in\left(0, s_{0}\right) \times(0, \mathcal{T}), \mathcal{T}=\frac{b}{c}$. Since 


$$
\underline{M}\left(s_{0}, t\right) \leq a+\gamma s_{0} \leq \underline{M}_{1}\left(s_{0}\right) \leq M\left(s_{0}, t\right)
$$

for each $t$ whenever $\underline{M}(s, t)$ and $M(s, t)$ make sense, the function $\underline{M}$ is a subsolution of an auxiliary problem on $\left(0, s_{0}\right) \times(0, \mathcal{T})$

$$
\begin{aligned}
\tilde{M}_{t} & =4 s \tilde{M}_{s s}+\frac{1}{\pi} \tilde{M} \tilde{M}_{s} \\
\tilde{M}(s, 0) & =M_{0}(s), \quad \tilde{M}\left(s_{0}, t\right)=\underline{M}_{1}\left(s_{0}\right) \leq M\left(s_{0}, t\right) .
\end{aligned}
$$

By the uniqueness property for the problem on finite intervals, $M(s, t)=\tilde{M}(s, t)$ for each $(s, t) \in\left(0, s_{0}\right) \times(0, \mathcal{T})$. Therefore

$$
\lim _{s \rightarrow 0, t \nearrow \mathcal{T}} M(s, t) \geq \lim _{s \rightarrow 0, t \succ \mathcal{T}} \underline{M}(s, t)=a>8 \pi .
$$

Since $a>8 \pi$ can be arbitrarily close to $8 \pi$, we conclude that the jump of $M^{*}(t)$ at $t=\mathcal{T}$ is equal to $8 \pi$.

Continuation. A continuation of the solution $M$ past blow up time $t=T, T \leq \mathcal{T}$ is obtained in [14, Th. 3.11] by using a regularization of (1.1) which cuts the nonlinearity for small $s>0$, see (3.9)-(3.11) below. Then, the approximating solutions are shown to tend monotonically to a unique smooth function extending $M$ to $(0, \infty) \times(0, \infty)$, cf. [14, Th. 3.5].

That is, we consider for $\nu \in(0,1)$ and $(s, t) \in(0, \infty) \times(0, \infty)$ the problem

$$
\begin{aligned}
M_{\nu, t} & =4 s M_{\nu, s s}+\frac{1}{\pi} H(s-\nu) M_{\nu} M_{\nu, s}, \\
M_{\nu}(0, t) & =\widehat{M}-M_{\nu}(\infty, t)=0, \\
M_{\nu}(s, 0) & =M_{0}(s), \quad s \in(0, \infty),
\end{aligned}
$$

where $H$ denotes the Heaviside function $H(\sigma)=0$ for $\sigma<0, H(\sigma)=1$ for $\sigma \geq 0$. It is rather standard to obtain a unique global in time solution $M_{\nu}$ of (3.9)-(3.11) since this problem is linear for $s \in(0, \nu)$, and for $s \in(\nu, \infty)$ is nonlinear but with a uniform diffusion. The solution $M_{\nu}$ satisfies $M_{\nu} \in \mathcal{C}^{1}((0, \infty) \times(0, \infty))$. Since the nonlinearity is discontinuous, this solution may have a jump of the second derivative $M_{\nu, s s}$ at $s=\nu$, but $M_{\nu}$ is continuous up to the boundary.

Since the problems for $M_{\nu}$ are uniformly parabolic in each rectangle $(\delta, \Delta) \times(\tau, T)$, $0<\delta<\Delta<\infty, 0<\tau<T<\infty$, and have smooth coefficients when $0<\nu<\delta$, the Schauder estimate

$$
\left\|M_{\nu}\right\|_{\mathcal{C}_{s, t}^{2+\alpha, 1+\alpha}([\delta, \Delta] \times[\tau, T])} \leq C(\alpha, \delta, \Delta, \tau, T)
$$

is verified for all $0<\nu<\delta$. Moreover, the solutions $M_{\nu}$ enjoy the following monotonicity property

$$
\text { for each } 0<\nu<\bar{\nu}<1 \quad M_{\nu}(s, t) \geq M_{\bar{\nu}}(s, t) .
$$

Indeed, if $\mathcal{L}_{\nu} w=w_{t}-4 s w_{s s}-\frac{1}{\pi} H(s-\nu) w w_{s}$, then $\mathcal{L}_{\nu} M_{\bar{\nu}} \leq 0$ and $\mathcal{L}_{\bar{\nu}} M_{\nu} \geq 0$ are satisfied, and since the comparison principle is valid for the approximating problems (3.9)-(3.11), the property (3.12) holds.

Thus, the family of functions tends to a limit $M$ monotonically as $\nu \searrow 0$, and by an argument of Ascoli-Arzelà type, $M \in \mathcal{C}_{s, t}^{2,1}((0, \infty) \times(0, \infty))$. It is standard to check that 
$M$ satisfies the equation (1.1), the initial condition (1.3) and $M(\infty, t)=\widehat{M}$. However, $M^{*}(t) \geq 0$ is not necessarily 0 , and in fact $M^{*}(t)$ strictly increases from $8 \pi$ to $\widehat{M}$ as $T<t \nearrow \infty$.

Uniqueness of continuation. The uniqueness is proved in a similar way as was before in Proposition 2.1, cf. also [14, Th. 3.5]. If $M$ and $\bar{M}$ are two functions $M, \bar{M} \in$ $\mathcal{C}\left([0, \infty), L_{\text {loc }}^{2}(0, \infty)\right) \cap \mathcal{C}_{s, t}^{2,1}((0, \infty) \times(0, \infty))$ satisfying the equation (1.1) pointwise in $(0, \infty) \times(0, \infty), M(\infty, t)=\bar{M}(\infty, t)=\widehat{M}$, and $M(s, 0)=\bar{M}(s, 0), M(s, t) \geq 8 \pi$, $\bar{M}(s, t) \geq 8 \pi$ for $s \in(0, \infty), t \in(T, \infty)$, then $N=M-\bar{M}$ solves the problem

$$
\begin{aligned}
N_{t} & =\frac{\partial}{\partial s}\left(4 s N_{s}+\frac{1}{2 \pi} N(M+\bar{M}-8 \pi)\right), \\
N(\infty, t) & =0 .
\end{aligned}
$$

Moreover, by the uniqueness of solutions $M, \bar{M}$ before blow up time $T$ and the continuity of $M, \bar{M} \quad N(s, t)=0$ for all $s \in(0, \infty)$ and $t \leq T$. Multiplying (3.13) by $s \Phi_{\delta}^{\prime}(N)$ analogously as in the proof of Proposition 2.1 we obtain

$$
\begin{aligned}
\frac{\mathrm{d}}{\mathrm{d} t} \int_{0}^{\infty} s \Phi_{\delta}(N) d s= & \left.4 s^{2} N_{s} \Phi_{\delta}^{\prime}(N)\right|_{0} ^{\infty}+\left.\frac{1}{2 \pi} s \Phi_{\delta}^{\prime}(N) N(M+\bar{M}-8 \pi)\right|_{0} ^{\infty} \\
& -\int_{0}^{\infty} 4 s N_{s}\left(s \Phi_{\delta}^{\prime}(N)\right)_{s} d s-\frac{1}{2 \pi} \int_{0}^{\infty} N(M+\bar{M}-8 \pi)\left(s \Phi_{\delta}^{\prime}(N)\right)_{s} \mathrm{~d} s \\
= & -\int_{0}^{1} 4 s^{2} \Phi_{\delta}^{\prime \prime}(N) N_{s}^{2} d s-\int_{0}^{\infty} 4 s\left(\Phi_{\delta}(N)\right)_{s} \mathrm{~d} s \\
& -\frac{1}{2 \pi} \int_{0}^{\infty} s \Phi_{\delta}^{\prime \prime}(N) N_{s} N(M+\bar{M}-8 \pi) \mathrm{d} s \\
& -\frac{1}{2 \pi} \int_{0}^{\infty} N(M+\bar{M}-8 \pi) \Phi_{\delta}^{\prime}(N) \mathrm{d} s
\end{aligned}
$$

since $N$ is bounded. Proceeding as in the proof of Proposition 2.1 we arrive in the limit $\delta \rightarrow 0$ at

$$
\frac{\mathrm{d}}{\mathrm{d} t} \int_{0}^{\infty} s|N(s, t)| d s \leq-\frac{1}{2 \pi} \int_{0}^{\infty}|N|(M+\bar{M}-16 \pi) \mathrm{d} s \leq 0
$$

because $M(s, t), \bar{M}(s, t) \geq 8 \pi$ for each $s>t, t \geq T$, as it has been obtained in the proof of blow up. Thus, the continuation of the solution is unique.

Asymptotic behavior for $t \rightarrow \infty$. We prove that for $\widehat{M}>8 \pi$ the concentration of whole mass at the origin takes place in the infinite time: $\lim _{t \rightarrow \infty} M^{*}(t)=\widehat{M}$. Here we recall the argument in [14, Th. 4.4] involving the regularizations (3.9)-(3.11) of the problem (1.1)-(1.3) used also to construct a continuation of $M$ past blow up time $T$.

It was proved in [14, Prop. 4.2] that any solution of (3.9)-(3.11) tends to the stationary solution $m_{\nu}$ as $t \rightarrow \infty$. These stationary solutions satisfy the relations

$$
m_{\nu}(s)=d \frac{s}{\nu} \text { for } s \in(0, \nu), \quad \frac{\widehat{M}-m_{\nu}(s)}{\widehat{M}+m_{\nu}(s)-8 \pi}=c\left(\frac{s}{\nu}\right)^{1-\frac{\widehat{M}}{4 \pi}} \text { for } s \in(\nu, \infty),
$$

where $c=\frac{\widehat{M}-d}{\widehat{M}+d-8 \pi}, d^{2}=\widehat{M}(\widehat{M}-8 \pi)$. Indeed, after the integration of (3.9) for time 
independent $m_{\nu}$ we get

$$
4 s m_{\nu, s}+\frac{m_{\nu}\left(m_{\nu}-8 \pi\right)}{2 \pi}=\frac{\widehat{M}(\widehat{M}-8 \pi)}{2 \pi} \quad \text { for } \quad s \in(\nu, \infty) .
$$

Integrating once again and using the condition for the continuity of the derivative $m_{\nu, s}$ at $s=\nu$ we obtain

$$
\frac{d}{\nu}=\frac{1}{\nu}\left(\frac{\widehat{M}}{4 \pi}-1\right) \frac{1}{\frac{1}{\widehat{M}-d}+\frac{1}{\widehat{M}+d-8 \pi}} .
$$

Evidently, $m_{\nu} \in \mathcal{C}^{1}(0, \infty)$ and the second derivative $m_{\nu}^{\prime \prime}$ has a jump.

The idea of the proof in [14, Section 4] consists in the comparison of $M_{\nu}$ with the solutions $M_{\nu}^{+}, M_{\nu}^{-}$of the problems

$$
\begin{aligned}
M_{\nu, t}^{ \pm} & =4 s M_{\nu, s s}^{ \pm}+\frac{1}{\pi} H(s-\nu) M_{\nu}^{ \pm} M_{\nu, s}^{ \pm}, \\
M_{\nu}^{ \pm}(0, t) & =(\widehat{M} \pm \mu)-M_{\nu}^{ \pm}(\infty, t)=0, \\
M_{\nu}^{ \pm}(s, 0) & =m_{\nu}^{ \pm}(s) .
\end{aligned}
$$

The functions $m_{\nu}^{+}, m_{\nu}^{-}$are defined by

$$
\begin{aligned}
& m_{\nu}^{ \pm}=\sqrt{\widehat{M}_{ \pm}\left(\widehat{M}_{ \pm}-8 \pi\right)} \frac{s}{s_{ \pm}}, \quad s \in\left(0, s_{ \pm}\right), \\
& \frac{\widehat{M}_{ \pm}-m_{\nu}^{ \pm}(s)}{\widehat{M}_{ \pm}+m_{\nu}^{ \pm}(s)-8 \pi}=\frac{\widehat{M}_{ \pm}-\sqrt{\widehat{M}_{ \pm}\left(\widehat{M}_{ \pm}-8 \pi\right)}}{\widehat{M}_{ \pm}+\sqrt{\widehat{M}_{ \pm}\left(\widehat{M}_{ \pm}-8 \pi\right)}}\left(\frac{s}{s_{ \pm}}\right)^{1-\frac{\widehat{M}_{ \pm}}{4 \pi}}, \quad s \in\left(s_{ \pm}, \infty\right),
\end{aligned}
$$

with any fixed $0<s_{+}<\nu<s_{-}<\infty$. Thus, $m_{\nu}^{+}, m_{\nu}^{-}$are a supersolution, a subsolution, resp. of the stationary problem (3.9)-(3.10) with $\widehat{M}_{+}=\widehat{M}+\mu, \widehat{M}_{-}=\widehat{M}-\mu, \mu \in$ $(0, \widehat{M}-8 \pi)$, resp. The crucial observation from the comparison principle for the problems of the type (3.9)-(3.10) is that

$$
M_{\nu, t}^{-} \geq 0, \quad M_{\nu, t}^{+} \leq 0 .
$$

Indeed, this is a consequence of the properties

$$
\begin{aligned}
& 4 s M_{\nu, s s}^{-}+\frac{1}{\pi} H(s-\nu) M_{\nu}^{-} M_{\nu, s}^{-} \geq 0, \\
& 4 s M_{\nu, s s}^{+}+\frac{1}{\pi} H(s-\nu) M_{\nu}^{+} M_{\nu, s}^{+} \leq 0,
\end{aligned}
$$

resp. These properties imply

$$
\begin{aligned}
\liminf _{t \rightarrow \infty} M_{\nu}^{-}(s, t) & \geq \underline{m}(s), \\
\limsup _{t \rightarrow \infty} M_{\nu}^{+}(s, t) & \leq \bar{m}(s),
\end{aligned}
$$

resp., where the functions $\underline{m}, \bar{m}$ satisfy the relations

$$
\begin{aligned}
& \underline{m}(s)=\sqrt{\widehat{M}_{-}\left(\widehat{M}_{-}-8 \pi\right)} \frac{s}{\nu}, \quad s \in(0, \nu), \\
& \frac{\widehat{M}_{-}-\underline{m}(s)}{\widehat{M}_{-}+\underline{m}(s)-8 \pi}=\frac{\widehat{M}_{-}-\sqrt{\widehat{M}_{-}\left(\widehat{M}_{-}-8 \pi\right)}}{\widehat{M}_{-}+\sqrt{\widehat{M}_{-}\left(\widehat{M}_{-}-8 \pi\right)}}\left(\frac{s}{\nu}\right)^{1-\frac{\widehat{M}_{-}}{4 \pi}}, \quad s \in(\nu, \infty),
\end{aligned}
$$


and

$$
\begin{aligned}
& \bar{m}(s)=\sqrt{\widehat{M}_{+}\left(\widehat{M}_{+}-8 \pi\right)} \frac{s}{\nu}, \quad s \in(0, \nu), \\
& \frac{\widehat{M}_{+}-\bar{m}(s)}{\widehat{M}_{+}+\bar{m}(s)-8 \pi}=\frac{\widehat{M}_{+}-\sqrt{\widehat{M}_{+}\left(\widehat{M}_{+}-8 \pi\right)}}{\widehat{M}_{+}+\sqrt{\widehat{M}_{+}\left(\widehat{M}_{+}-8 \pi\right)}}\left(\frac{s}{\nu}\right)^{1-\frac{\widehat{M}_{+}}{4 \pi}}, \quad s \in(\nu, \infty),
\end{aligned}
$$

Finally, we take the limit $\mu \rightarrow 0$ in (3.18)-(3.19), and we get $\liminf _{t \rightarrow \infty} M(s, t) \geq \widehat{M}$ because $M(s, t) \geq M_{\nu}(s, t)$. Now, for a fixed $s>0$ taking the limit as $\nu \rightarrow 0$ leads to

$$
\frac{\widehat{M}-M}{\widehat{M}+M-8 \pi} \rightarrow 0 \text {. }
$$

Therefore $M_{\nu}(s) \rightarrow \widehat{M}$ and, of course, $0 \leq M_{\nu}(s) \leq \widehat{M}$. As it concerns $M_{\nu}(s, t)$, it is important that this quantity is monotone with respect to $t$.

To conclude the proof, we will show that (after [14, Th. 4.4])

$$
\lim _{t \rightarrow \infty} M^{*}(t)=\widehat{M} \text {. }
$$

The maximum principle gives $M_{s}(s, t)>0$ for all $s>0, t>0$, hence $M^{*}(t)<\widehat{M}$ for each $t \geq 0$.

Let $\underline{M}(s, t)$ be the global in time solution of the problem (1.1) with smaller mass than $\widehat{M}: \underline{M}(0, t) \geq 0, \underline{M}(\infty, t)=\widehat{M}-\mu(\mu \in(0, \widehat{M}-8 \pi))$, and $\underline{M}(s, 0)=(\widehat{M}-\mu) \frac{s}{s+b}, b>0$. This initial condition is a stationary subsolution:

$$
4 s \underline{M}_{s s}(s, 0)+\frac{1}{\pi} \underline{M}(s, 0) \underline{M}_{s}(s, 0) \geq 0 .
$$

Therefore, $\underline{M}_{t} \geq 0$ for all $s>0, t>0$, and as a consequence

$$
\frac{\partial}{\partial s}\left(4 s \underline{M}_{s}+\frac{1}{2 \pi}\left(\underline{M}^{2}-8 \pi \underline{M}\right)\right) \geq 0
$$

for all $s>0, t>0$.

Now, $A=\lim _{s \rightarrow 0} s \underline{M}_{s}(s, t)$ exists, and since

$$
\frac{1}{\varepsilon} \int_{0}^{\varepsilon} s \underline{M}(s, t) \mathrm{d} s=\underline{M}(\varepsilon, t)-\frac{1}{\varepsilon} \int_{0}^{\varepsilon} \underline{M}(s, t) \mathrm{d} s \rightarrow 0
$$

as $\varepsilon \rightarrow 0$, the equality $A=0$ follows. Thus,

$$
\frac{\mathrm{d}}{\mathrm{d} t} \int_{0}^{1} \underline{M}(s, t) \mathrm{d} s \geq \frac{1}{2 \pi}\left(\underline{M}^{2}-8 \pi \underline{M}\right),
$$

and, again by the monotonicity in $t \geq 0$,

$$
\liminf _{t \rightarrow \infty} \frac{\mathrm{d}}{\mathrm{d} t} \int_{0}^{1} \underline{M}(s, t) \mathrm{d} s \geq \frac{\widehat{M}-\mu-8 \pi}{2 \pi}\left(\widehat{M}-\mu-\lim _{t \rightarrow \infty} \underline{M^{*}}(t)\right) \geq 0 .
$$

Then $\lim _{t \rightarrow \infty} \underline{M^{*}}(t) \geq \widehat{M}-\mu$ follows.

Given $\mu>0$, there exists $b>0$ large enough so that $M(s, 1) \geq \underline{M}(s, 0)=(\widehat{M}-\mu) \frac{s}{s+b}$ and $M(s, t+1) \geq \underline{M}(s, t)$ for $s>0, t>0$. The relation $\liminf _{t \rightarrow \infty} \underline{M^{*}}(t) \geq \widehat{M}-\mu$ is a consequence of the above. Passing to the limit $\mu \rightarrow 0$, the proof is finished: whole mass concentrates at the origin in infinite time. 
Acknowledgements. This work, motivated by numerous conversations with Tadeusz Nadzieja, owes much to the cooperation and discussions with Jan Goncerzewicz, Philippe Laurençot and Grzegorz Karch. The preparation of this paper was partially supported by the European Commission Marie Curie Host Fellowship for the Transfer of Knowledge "Harmonic Analysis, Nonlinear Analysis and Probability" MTKD-CT-2004-013389 and by the Polish Ministry of Science project N201 022 32/0902.

\section{References}

[1] P. Biler, The Cauchy problem and self-similar solutions for a nonlinear parabolic equation, Studia Math. 114 (1995), 181-205.

[2] P. Biler, Existence and nonexistence of solutions for a model of gravitational interaction of particles. III, Colloq. Math. 68 (1995), 229-239.

[3] P. Biler, Growth and accretion of mass in an astrophysical model, Appl. Math. (Warsaw) 23 (1995), 179-189.

[4] P. Biler, D. Hilhorst and T. Nadzieja, Existence and nonexistence of solutions for a model of gravitational interaction of particles. II, Colloq. Math. 67 (1994), 297-308.

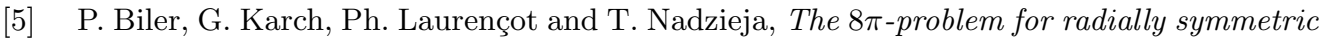
solutions of a chemotaxis model in a disc, Topol. Methods Nonlinear Anal. 27 (2006), 133-144.

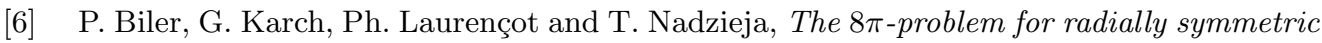
solutions of a chemotaxis model in the plane, Math. Methods Appl. Sciences 29 (2006), 1563-1583.

[7] P. Biler and T. Nadzieja, Growth and accretion of mass in an astrophysical model. II, Appl. Math. (Warsaw) 23 (1995), 351-361.

[8] P. Biler and T. Nadzieja, A nonlocal singular parabolic problem modelling gravitational interaction of particles, Adv. Differential Equations 3 (1998), 177-197.

[9] A. Blanchet, J. Dolbeault and B. Perthame, Two dimensional Keller-Segel model: Optimal critical mass and qualitative properties of the solutions, Electron. J. Diff. Eqns. 2006, 44, $1-33$.

[10] W. Jäger and S. Luckhaus, On explosions of solutions to a system of partial differential equations modelling chemotaxis, Trans. Amer. Math. Soc. 329 (1992), 819-824.

[11] O. A. Ladyženskaja, V. A. Solonnikov and N. N. Ural'ceva, Linear and Quasilinear Equations of Parabolic Type, Transl. Math. Monogr. 23, Amer. Math. Soc., Providence, RI, 1988.

[12] T. Nagai, Blow-up of radially symmetric solutions to a chemotaxis system, Adv. Math. Sci. Appl. 5 (1995), 581-601.

[13] M. Nagasawa, Schrödinger Equations and Diffusion Theory, Birkhäuser, Basel, Boston, Berlin, 1993.

[14] M. Primicerio and B. Zaltzman, Global in time solution to the Keller-Segel model of chemotaxis, 1-18, preprint 2003.

[15] T. Senba and T. Suzuki, Weak solutions to a parabolic-elliptic system of chemotaxis, J. Funct. Anal. 191 (2002), 17-51.

[16] T. Suzuki, Free Energy and Self-Interacting Particles, PNLPDE 62, Birkhäuser, Boston, 2005 . 